\title{
A large animal neuropathic pain model in sheep: a strategy for improving the predictability of preclinical models for therapeutic development
}

This article was published in the following Dove Press journal: Journal of Pain Research

24 October 2012

Number of times this article has been viewed

\author{
Denise Wilkes' \\ Guangwen $\mathrm{Li}^{2}$ \\ Carmina F Angeles ${ }^{3}$ \\ Joel T Patterson ${ }^{4}$ \\ Li-Yen Mae Huang ${ }^{2}$ \\ 'Department of Anesthesiology, \\ ${ }^{2}$ Department of Neuroscience \\ and Cell Biology, ${ }^{3}$ Department of \\ Neurosurgery University of Texas \\ Medical Branch, Galveston, TX, USA; \\ ${ }^{4}$ Neurospine Institute, Eugene, \\ OR, USA
}

Background: Evaluation of analgesics in large animals is a necessary step in the development of better pain medications or gene therapy prior to clinical trials. However, chronic neuropathic pain models in large animals are limited. To address this deficiency, we developed a neuropathic pain model in sheep, which shares many anatomical similarities in spine dimensions and cerebrospinal fluid volume as humans.

Methods: A neuropathic pain state was induced in sheep by tight ligation and axotomy of the common peroneal nerve. The analgesic effect of intrathecal (IT) morphine was investigated. Interspecies comparison was conducted by analyzing the ceiling doses of IT morphine for humans, sheep, and rats.

Results: Peroneal nerve injury (PNI) produced an $86 \%$ decrease in von-Frey filament-evoked withdrawal threshold on postsurgery day 3 and the decrease lasted for the 8 -week test period. Compared to the pre-injury, sham, and contralateral hindlimb, the IT morphine dose that produces $50 \%$ of maximum analgesia $\left(\mathrm{ED}_{50}\right)$ for injured PNI hindlimb was 1.8 -fold larger and $\mathrm{E}_{\max }$, the dose that produces maximal analgesia, was 6.1-fold lower. The sheep model closely predicts human IT morphine ceiling dose by allometric scaling. This is in contrast to the approximately 10 -fold lower morphine ceiling dose predicted by the rat spinal nerve ligated or spared nerve injury models.

Conclusion: PNI sheep model has a fast onset and shows stable and long-lasting pain behavioral characteristics. Since the antinociceptive properties of IT morphine are similar to those observed in humans, the PNI sheep model will be a useful tool for the development of analgesics. Its large size and consistent chronic pain behavior will facilitate the development and evaluation of surgical intervention and gene therapy. The PNI sheep pain model provides us with the opportunity for multi-species testing, which will improve the success of clinical trials.

Keywords: interspecies drug scaling, neuropathic pain model, ovine, ovine pain model

\section{Introduction}

Chronic neuropathic pain is estimated to affect between $3.3 \%$ and $8.2 \%$ of the general population and is a major health problem. ${ }^{1}$ Pain management remains difficult, and analgesia is often unsatisfactory. Most studies of neuropathic pain use rodent models to determine pain pathways, assess mechanisms of chronic pain and discover potential new therapeutic targets. ${ }^{2}$ The major shortcoming of these models is the frequent failure to predict drug efficacy in clinical trials. ${ }^{3}$ New strategies to improve neuropathic pain treatment are needed. This study introduces a large animal chronic neuropathic pain model that provides consistent evoked pain behavior and improves the predictability of drug efficacy.

There are several rodent neuropathic models that incorporate one or multiple peripheral nerve injuries such as ligation of the L5 and L6 spinal nerves (SNL), loose
Correspondence: Li-Yen Mae Huang Department of Neuroscience and Cell Biology, University of Texas Medical Branch, 30I University Blvd, Galveston, TX 77555, USA

Tel +I 4097726555

Fax +I 409772 I826

Email Imhuang@utmb.edu 
ligature of the sciatic nerve, and ligation of the peroneal and tibial nerves in the spared nerve injury (SNI) model. ${ }^{4-6}$ After nerve injury, the animal develops mechanical allodynia in the paw. Advantages of these models are a known onset, reproducible paw withdrawal thresholds, and duration. Disadvantages of the rodent models are the large size difference between rodents and humans, which often introduces significant scale-up errors in drug dose estimation, a lack of genetic diversity, and a relatively short lifespan.

We developed a nerve injury pain model in sheep involving tight ligation and axotomy of the common peroneal nerve. This model is similar to rodent SNI models ${ }^{4,7}$ except that only the common peroneal nerve is injured. A clear advantage of using a large animal model such as sheep is the similarity in body size to humans. This allows us to use clinical sensory testing equipment and drug delivery devices. The smaller weight difference between sheep and humans helps us better approximate clinical doses, reduces errors in scale-up, and can be used to determine doses in drugs not amenable to allometric scaling. In addition, sheep share several significant similarities with humans including similar spine dimensions, cerebrospinal fluid volume, and cardiac and pulmonary functional parameters. ${ }^{89}$ Another benefit of using sheep for chronic pain models is their genetic diversity. A worldwide analysis of sheep genomes showed that the modern-day sheep retains more genetic variability than other domesticated animals ${ }^{10}$ and therefore will allow testing in a diverse population. The sheep also has a longer lifespan (approximately 20 years) compared to the rat (2-4 years), which is beneficial for chronic pain models. ${ }^{11}$ Together, these advantages provide additional guidance in designing successful chronic pain therapies such as intrathecal (IT) drug and gene therapy trials.

Sheep have been used extensively in research to study orthopedic spine hardware, IT drug delivery, and treatments for acute pain. ${ }^{8,12,13}$ Sheep models have provided useful guidelines for acute pain treatments. Early studies by Lebeaux demonstrated a method by which to assess pain and sensation to mechanical stimuli produced by forceps pinch in restrained sheep. ${ }^{14}$ Later studies by Nolan et al ${ }^{13}$ quantified acute pain behavior using a fabricated force-calibrated pneumatic pin device. Other researchers have used Nolan's models to study the effects of $\alpha_{2}$ agonists, opioids, and NSAIDs. ${ }^{15,16}$ This model required researchers to fabricate their own testing devices, which may have increased variability among studies. Nevertheless, these studies served as pre-clinical trials for successful clinical therapies for postoperative pain and validation for the safety for chronic IT opioid therapy. ${ }^{17,18}$
In contrast, large animal chronic pain studies have been limited. Studies of sheep foot rot described a chronic inflammatory pain condition and improved veterinary treatment. ${ }^{19,20}$ Other sheep studies have provided safety guidelines for clinical use of IT opioid therapy. ${ }^{8,18}$

Sheep are ideally suited as behavior models. The docile nature of the sheep allows relatively easy handling and training. Sheep also tolerate repetitive behavioral testing without changes in behavior or signs of stress. On the practical side, sheep are readily available and economical, and their use is more ethically acceptable than that of nonhuman primates.

The objectives of this study were to describe the behavior of pre-injury or naïve sheep, characterize the development of pain behavior in sheep after peroneal nerve injury (PNI), and compare analgesia of IT morphine among pre-injury, sham, and PNI sheep. Quantitative analysis of morphine analgesia provided a validation of the sheep model and facilitated interspecies comparison between human, sheep, and rat. The sheep model will likely be a valuable tool in drug development for neuropathic pain.

\section{Materials and methods}

\section{Animals}

Sheep were purchased from Talley Ranch (Vualde, TX). Female merino sheep weighing 30-40 kg and approximately 3-4 years old were used for the peripheral nerve injury sheep model. Female sheep were used for this study as they were easier to train and more docile than male sheep. Sheep were tested over 2 months in order to measure the reproducibility of the behavior test over time. Female sheep showed stable behavioral characteristics without significant changes over the month, indicating that the estrous cycle did not affect the behavior test. Upon arrival, sheep were examined by a veterinarian and deemed nonpregnant and free from disease. All experimental procedures were approved by the Institutional of Animal Care and Use Committee at the University of Texas Medical Branch and are in accordance with the guidelines of National Institutes of Health and of the International Association for the study of Pain (IASP). Twenty sheep were used for the study. After surgery, the sheep were inspected for infection and tested for motor function change by examination of the passive and active motion of the limbs. Two sheep developed an infection at the surgical site. Another sheep developed motor weakness after surgery. These three sheep were euthanized. The remaining 17 sheep did not exhibit any clinical symptoms for the duration of the study. All experimental animals were maintained in good health. The animals were housed in solitary cages 
due to the concern that resident sheep would manipulate the IT catheters.

\section{Anesthesia}

Sheep were fasted for 24 hours prior to surgery. The preanesthetic preparation included intramuscular (IM) application of ketamine $(600 \mathrm{mg})$ and buprenorphine $(0.3 \mathrm{mg})$. An intravenous (IV) line was then placed in the external jugular and the sheep received an additional $200 \mathrm{mg}$ ketamine IV and a prophylactic dose of antibiotic cefazolin (1 g, IV). After intubation, the sheep were maintained with isoflurane $(2 \%)$ anesthesia and mechanically ventilated. The sheep received IV fluids ( $0.9 \%$ normal saline). Body temperature was maintained with a circulating water pad. Oxygen saturation was monitored and maintained between $98 \%-100 \%$. Heart rate was maintained within $80-120$ beats per minute. Systolic pressure was maintained within $80-120 \mathrm{mmHg}$ and diastolic pressure was within $60-80 \mathrm{mmHg}$.

\section{Surgeries}

\section{IT catheter placement surgery}

Sheep were positioned prone with hindlimbs cephalad beside the abdomen. The lumbrosacral area was sterilely prepped. A vertical midline incision was made over the L-6 to S-2 vertebrae. The dura was exposed and nicked. An epidural catheter (Radio-opaque Epidural Catheter; Epimed, Johnstown, NY) was placed into the subarachnoid space and advanced until the $10 \mathrm{~cm}$ mark was at the opening of the dura. Once cerebrospinal fluid (CSF) flow through the catheter was confirmed, the dura was pulled close to the catheter with a 6-0 nylon suture and the catheter was secured to the muscle fascia. The dura, muscle fascia, and skin were then closed. The catheter was secured subcutaneously, tunneled to the cervical region, and secured to the skin of the sheep with sutures.

\section{Common peroneal nerve injury surgery}

The common PNI was performed while the animal was under general anesthesia for placement of the IT catheter. The sheep's left hindlimb was repositioned laterally and sterilely prepared and draped. A vertical incision was made mid-thigh and the common peroneal nerve and tibial nerve were exposed. The common peroneal nerve was ligated with 2-0 silk sutures and cut distally. A $1 \mathrm{~cm}$ segment was removed from the distal stump to prevent regrowth. Fascia and skin were closed. The analgesia buprenorphine was administered IM at a dose of $0.005-0.01 \mathrm{mg} / \mathrm{kg}$ prior and every 12 hours after surgery for 2 days postoperatively. Behavioral testing was begun on the third day after surgery.

\section{Behavioral analysis \\ Hindlimb withdrawal threshold test}

To obtain consistent results, the sheep were conditioned prior to their use in experiments and one examiner performed all behavioral testing. Hindlimb withdrawal thresholds were measured using a stiff von Frey filament tip (1000 g) attached to an anesthesiometer (model 2390, IITC, Inc; Life Science Instruments, Woodland Hills, CA). The stiff tip was applied to the test area on the hindlimb with increasing pressure until the hindlimb withdrew. The pressure prior to the loss of contact between the tip and the hindlimb was displayed and recorded on the meter.

\section{Effect of IT morphine on hindlimb withdrawal threshold}

Baseline withdrawal thresholds were determined prior to morphine administration. Morphine (Baxter Healthcare Co, Deerfield IL) was diluted in $0.9 \%$ normal saline and a constant volume of $100 \mu \mathrm{L}$ for each dose was maintained. After verification of CSF aspiration, the morphine solution was injected into the IT catheter. The catheter was then flushed with $100 \mu \mathrm{L} 0.9 \%$ normal saline. For the morphine time course, the withdrawal threshold was measured with the anesthesiometer at 15- or 30-minute intervals following an IT injection of a dose of morphine. A cumulative morphine dose-response protocol ${ }^{21}$ was used to determine the morphine dose-dependent effect on hindlimb withdrawal threshold. Morphine was given through the IT catheter. Thirty minutes later, the withdrawal threshold was measured. A subsequent dose of morphine was then applied. The procedure was repeated 30 minutes later until two to four ascending doses of morphine had been applied.

\section{Statistical analysis}

Animals were randomly assigned to each treatment group. Results were expressed as mean \pm standard error of the mean. Changes in withdrawal thresholds of ipsilateral and contralateral hindlimbs of pre-injury, sham, and PNI sheep were analyzed using one-way analysis of variance (ANOVA) followed by Bonferroni correction. Comparisons of the time course change in withdrawal thresholds of ipsilateral and contralateral hindlimbs following sham and PNI surgery were analyzed using mixed ANOVA. The time course of morphine effect within one sheep group was analyzed using one-way ANOVA repeated measures followed by post hoc paired $t$-tests with Bonferroni correction. Dose-response curves for morphine were plotted as mean withdrawal threshold versus cumulative morphine dose. The cumulative morphine doses 
were calculated using the morphine dose decay factor, which were obtained from the morphine time course plot. In our study, the dose decay factor was 1.0 after 30 minutes, 1.0 after 60 minutes, and 0.60 after 90 minutes of morphine application. For example, the cumulative morphine dose after injection of $2,50,200$, and $500 \mu \mathrm{g} / 100 \mu \mathrm{L}$ at 30 -minute intervals was $2 \times 0.0+50 \times 0.6+200 \times 1.0+500 \times 1.0=730.0 \mu \mathrm{g} / 100 \mu \mathrm{L}$. The dose decay factor for $2 \mu \mathrm{g}$ morphine was set to 0 because the analgesic effect of low-dose morphine $(<10 \mu \mathrm{g})$ dissipated 120 minutes after its application as measured in this study. Theoretical curves were obtained by fitting the experimental data with the Hill equation (KaleidaGraph 4.0; Synergy Software, Reading, PA). The effective dose producing $50 \%$ of the analgesic effect or potency $\left(\mathrm{ED}_{50}\right)$ and the maximal analgesic efficacy $\left(\mathrm{E}_{\mathrm{max}}\right)$ were determined from the fitted curve.

\section{Results}

\section{Conditioning}

Sheep are herding animals and prefer to congregate with other sheep, maintaining verbal and physical contact. In addition, sheep are prey animals. The native behavior of the sheep is to flee upon sight, smell, or sound of a predator. Prior to conditioning, the sheep avoided contact with the examiner by stepping away or stomping its forelimbs. All sheep underwent conditioning. The conditioning protocol involved placement of the sheep in a stanchion (Figure 1A) and transport to a testing room. The environment consisted of two to three stanchions, a room with its door closed, and an examiner who performed the behavioral testing. Thirty minutes later, the sheep was returned to solitary housing. The conditioning protocol was repeated for three consecutive days. To obtain consistent results, the conditioning protocol and the criteria for the testing environment were strictly followed.

Following the conditioning, the sheep tolerated the presence and touch of the examiner, thus allowing access to test site and IT catheters. For behavioral testing, each sheep was in its own metabolic stanchion but within sight of the other sheep. The sheep tolerated the stanchion better if there were multiple sheep in stanchions in the room. We observed no change in the sheep sensitivity response due to the presence of other sheep.

\section{Pre-injury sheep behavioral assessment}

For behavioral testing, several sheep, each in its own stanchion, were transported to the testing room. The sheep were calm within 30 minutes and were ready to undergo a hindlimb threshold test. A stiff von Frey filament tip attached to an anesthesiometer was applied with increasing pressure to the cutaneous area innervated by the sural nerve on the hindlimb (Figure 1B) of the sheep for 3 seconds. A withdrawal of the hindlimb within 3 seconds of application was considered a positive response. The sheep returned to a resting state within 10 seconds. The resting state involves the sheep looking forward and standing, exhibiting nonstressed behaviors such as chewing. The stiff tip application was repeated three times with a 30-second wait period between applications; these measurements were averaged. Withdrawal thresholds of ipsilateral and contralateral hindlimb prior to injury were not significantly different (pre-injury: ipsilateral $57.3 \pm 3.5 \mathrm{~g}$, $\mathrm{n}=6$ and contralateral $56.8 \pm 2.4 \mathrm{~g}, \mathrm{n}=6$ ) (Figure 2).

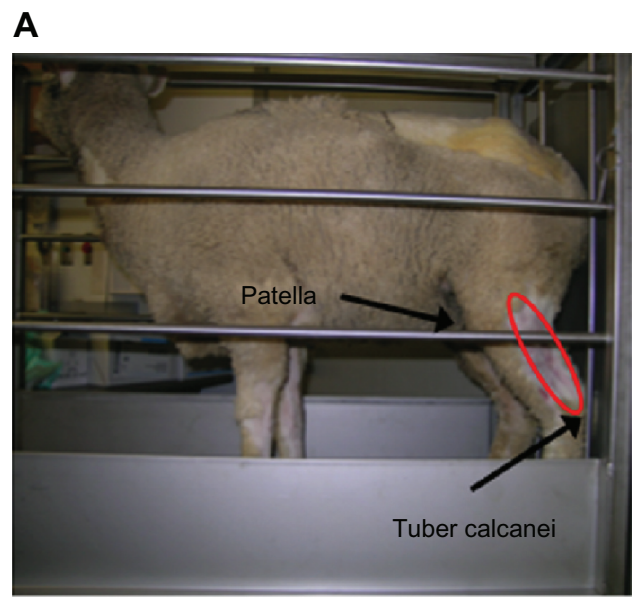

B

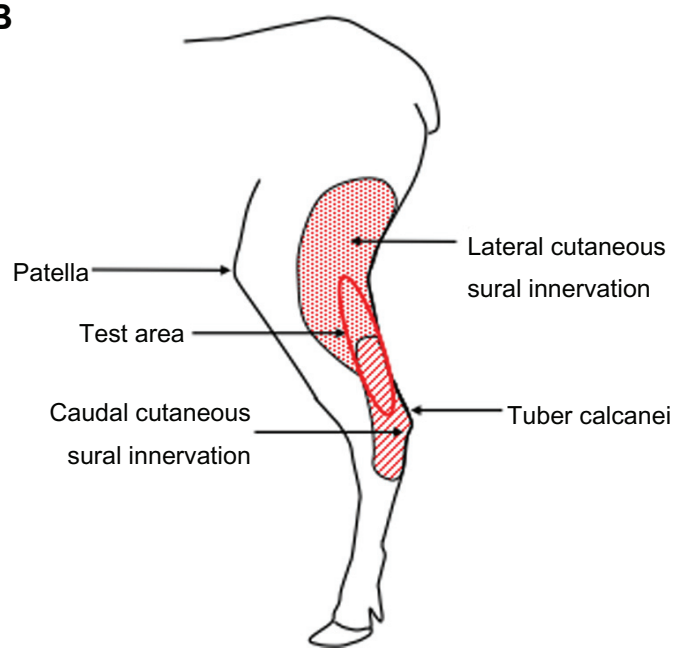

Figure I Behavioral test area in sheep. (A) Sheep in a stanchion. The sheep was able to move forward and backward within the limited space of the stanchion. The testing area (circled in red) is located below the patella and above the tuber calcanei. (B) Cutaneous innervation of the test area.

Notes: The cutaneous innervation of the sural nerve is located on the lateral side of the hindlimb. The test area (circled in red) is between the bony landmarks of the patella and tuber calcanei and within the areas of innervation of the lateral (shaded with dots) and caudal cutaneous (shaded with diagonal lines) sural nerves. 


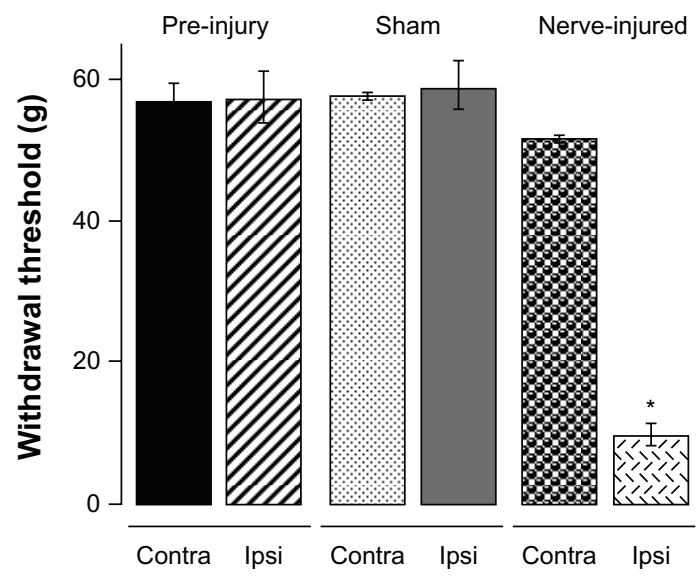

Figure 2 Sheep hindlimb withdrawal thresholds.

Notes: In pre-injury sheep, withdrawal thresholds were determined I-2 weeks prior to surgery $(n=6)$. There was no significant difference between ipsilateral and contralateral hindlimbs. In sham sheep, withdrawal thresholds of ipsilateral and contralateral sham hindlimbs, determined 3 weeks after sham surgery, were not significantly different $(n=3)$. Furthermore, there was no significant difference between sham and pre-injury sheep. In nerve injured sheep, withdrawal thresholds of ipsilateral and contralateral hindlimbs were measured 3 weeks after PNI surgery $(n=6)$. Data were analyzed using one-way ANOVA followed by Bonferron correction. The ipsilateral nerve injured hindlimb showed a markedly reduced threshold $(9.6 \pm 1.7 \mathrm{~g}, \mathrm{n}=6) ; * P<0.000 \mathrm{I}$. The withdrawal threshold of the contralateral hindlimb was not significantly different from sham or pre-injury sheep. Abbreviations: ANOVA, analysis of variance; PNI, peroneal nerve injury.

\section{Development of chronic neuropathic pain model in sheep}

Following pre-injury behavioral assessment, an IT catheter and sham/nerve injury surgery was performed on the sheep. For preinjury animal group, only IT catheter placement surgery was performed. Withdrawal threshold measurements commenced three days after surgery. The placement of IT catheters did not change withdrawal thresholds (data not shown).

In response to peroneal nerve injury, the sheep hoof curled inward (plantar flexion) as the result of weakness to extend the hoof. The sheep supported its weight on the joint proximal to the hoof (fetlock) and was able to withdraw the hindlimb, walk, run, and feed, similar to pre-surgery levels as determined by observations. The sham sheep did not exhibit any hoof deformity. Because of the presence of the physical deformity only in the nerve-injured group, the investigator was not blinded during behavioral measurements.

Three weeks after PNI surgery, the withdrawal threshold of the ipsilateral limb was significantly reduced $(9.6 \pm 1.7 \mathrm{~g}$, $\mathrm{n}=6$ ). PNI sheep developed allodynia, ie, hindlimb withdrawal in response to a stimulus not normally painful or less than the pre-injury threshold (57 g). The withdrawal threshold in the contralateral hindlimb of PNI sheep $(57.4 \pm 3.4 \mathrm{~g})$ was not significantly different from that in the sham or pre-injury sheep.

Development of allodynia pain was studied in detail. Withdrawal thresholds of sham and PNI sheep were measured on day 3 after the surgery and then at weekly intervals over an 8-week test period (Figure 3 ). There were no significant differences in withdrawal thresholds between the ipsilateral or contralateral hindlimb of sham sheep or between sham and pre-injury sheep. Compared with pre-injury sheep, the withdrawal threshold of the ipsilateral hindlimb of PNI sheep was reduced by approximately $86 \%$ from $57.3 \pm 2.4 \mathrm{~g}$ prior to surgery to $9.6 \pm 1.7 \mathrm{~g}(\mathrm{n}=6)$ on postsurgery day 3 .
A

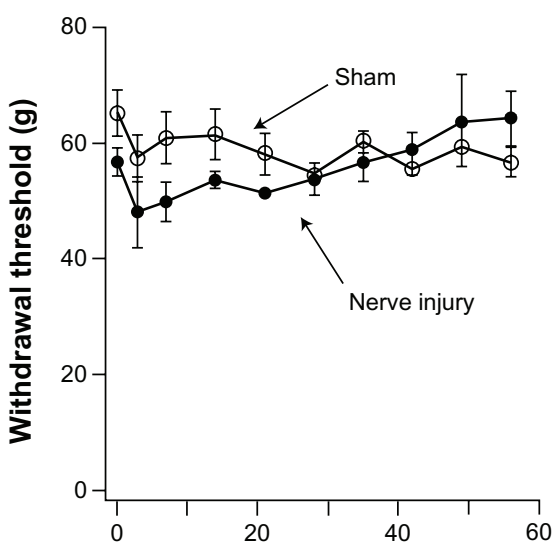

B

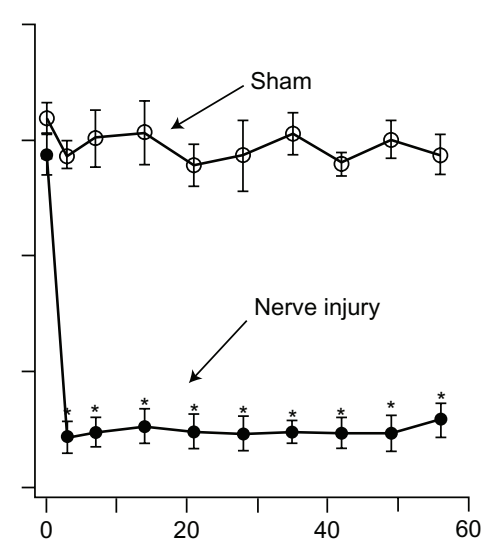

Days after nerve injury

Figure 3 Development of prolonged allodynia after nerve injury. Hindlimb withdrawal thresholds were determined over the 8-week test period $(n=6)$. $(\mathbf{A})$ Withdrawal thresholds of the contralateral hindlimb showed no significant difference in sham and PNI sheep. (B) Withdrawal thresholds of the ipsilateral hindlimb of PNI sheep were significantly reduced 3 days after nerve injury and remained reduced for the 8 weeks of the study.

Notes: Withdrawal thresholds of the ipsilateral hindlimb of sham sheep were not significantly different from those of the contralateral hindlimb and remained unchanged for the duration of the study. Comparison of withdrawal thresholds among sheep groups was analyzed by mixed ANOVA. *Significant difference $(P<0.00 \mathrm{I})$ from day 0 . All of the experiments were repeated in six sheep.

Abbreviations: ANOVA, analysis of variance; PNI, peroneal nerve injury. 
The threshold reduction persisted for the entire duration of our study. Thus, PNI produces severe allodynia lasting for at least 8 weeks. The withdrawal threshold of the contralateral hindlimb of the PNI sheep was $51.5 \pm 0.6 \mathrm{~g}(\mathrm{n}=6)$, which was not significantly different from that of pre-injury $(58.3 \pm 3.5 \mathrm{~g}, \mathrm{n}=6)$ or sham sheep $(56.8 \pm 2.4 \mathrm{~g}, \mathrm{n}=3)$.

\section{Effect of morphine on pain behavior}

IT morphine analgesia was studied 3 weeks after IT tube placement. The IT application allowed morphine direct access to the dorsal root ganglia and spinal dorsal horn opioid receptors. At a saturating dose of IT morphine, the sheep responded briskly to mechanical stimulation and showed no sign of sedation or respiratory depression such as a lowered head position, decreased alertness, or a respiratory rate $(<10$ breaths/min).

The time course of analgesia, produced by various doses of IT morphine, was measured in pre-injury sheep (Figure 4). Following an injection of a dose of morphine, the withdrawal threshold was measured every 15 minutes for 120 minutes and then at 30 minute intervals thereafter. The onset of analgesia was observed within 15 minutes of the IT morphine dose. Peak analgesia was reached at 30 minutes and continued to 75 minutes after the morphine injection $(n=3)$. The analgesia started to decay at 90 minutes and returned to baseline level 150-200 minutes after the morphine application. The level

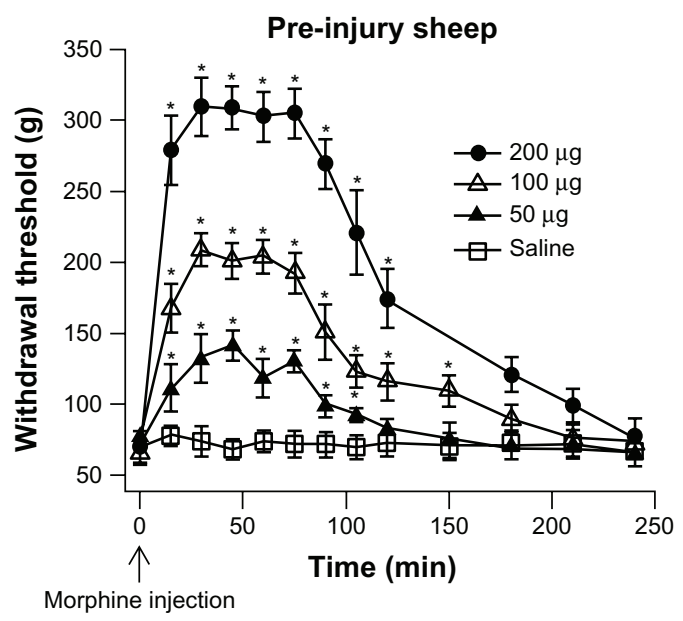

Figure 4 Time course of IT morphine effect.

Notes: The analgesia produced by a single dose of IT morphine was measured in pre-injury sheep $(n=3)$. A dose of morphine, eg, $50 \mu \mathrm{g} / 100 \mu \mathrm{L}$, was given at time 0 . The withdrawal thresholds were measured at various intervals. The analgesia could be observed within 15 minutes of morphine application and reached a peak value after 30 minutes. The analgesia was maintained at the peak level for another 45 minutes before beginning to decrease at 75 minutes after morphine application. The morphine analgesic effect dissipated in 150-200 minutes. The level of peak analgesia was dose-dependent. Data were analyzed by one-way ANOVA repeated measures followed by post hoc test using the Bonferroni method. *Significant change from the withdrawal threshold measured prior to morphine injection.

Abbreviations: ANOVA, analysis of variance; IT, intrathecal. of peak analgesia increased with the dose of morphine. The sheep did not exhibit changes in activity after administration of morphine such as jumping or startle response.

Dose-response curves of IT morphine in pre-injury, sham, and PNI sheep were determined. A cumulative drugdosing protocol was used with a starting dose and fixed interval. ${ }^{21}$ An initial dose of morphine was applied to the sheep. The withdrawal threshold was measured 30 minutes later and a second dose of morphine was then applied. The procedure was repeated 30 minutes later until four ascending doses of morphine, 2, 50, 200, and $500 \mu \mathrm{g} / 100 \mu \mathrm{L}$, had been applied. The animal was then returned to the cage and allowed to recover. Three to four days later, the effects of a set of different doses of morphine, 20, 100, and $300 \mu \mathrm{g} / 100 \mu \mathrm{L}$, were tested. In some cases, the effects of a third set of doses of morphine, 700 and $1000 \mu \mathrm{g} / 100 \mu \mathrm{L}$, were tested after another 3-4 days. Since the withdrawal thresholds prior to IT morphine administration measured on different testing days were not significantly different, the morphine effects had dissipated during the wait period (3-4 days). We observed no significant change in the effect of morphine dose-response on subsequent days of testing. This was determined by comparing the same IT morphine dose tested on different days.

A cumulative morphine dose-response curve was constructed according to the method described under "Statistical analysis" in the "Materials and methods" section (see also Figure 5). The $\mathrm{ED}_{50}$ and $\mathrm{E}_{\max }$ were obtained by fitting the experimental data with the Hill equation. The $\mathrm{ED}_{50}$ and $\mathrm{E}_{\max }$ of morphine for the contralateral hindlimbs of pre-injury $(73.4 \pm 3.3 \mu \mathrm{g}, 335.0 \pm 6.3 \mathrm{~g}, \mathrm{n}=6)$, sham $(74.8 \pm 13.0 \mu \mathrm{g}$, $362.4 \pm 15.3 .0 \mathrm{~g}, \mathrm{n}=3)$, and contralateral hindlimb of nerveinjured $(88.3 \pm 9.5 \mu \mathrm{g}, 336.7 \pm 10.7 \mathrm{~g}, \mathrm{n}=6)$ sheep were not significantly different. The $\mathrm{ED}_{50}$ and $\mathrm{E}_{\max }$ of morphine for the ipsilateral hindlimb of pre-injury $(76.3 \pm 11.9 \mu \mathrm{g}$, $361.0 \pm 15.2 \mathrm{~g}, \mathrm{n}=6)$ and sham sheep $(65.5 \pm 8.1 \mu \mathrm{g}$, $348.3 \pm 14.5 \mathrm{~g}, \mathrm{n}=3$ ) were not significantly different. In contrast, the $\mathrm{ED}_{50}$ of morphine for ipsilateral hindlimb of the PNI sheep was much larger $(159.6 \pm 32.4 \mu \mathrm{g})$ and the $\mathrm{E}_{\max }$ was significantly reduced $(54.8 \pm 3.1 \mathrm{~g})$. Compared to the preinjury, sham, and contralateral hindlimb, $\mathrm{ED}_{50}$ increased by 1.8-fold and $\mathrm{E}_{\max }$ decreased by 6.1-fold after nerve injury.

\section{Discussion}

In this study, we demonstrated that a large animal model of chronic neuropathic pain could be reliably produced. This model is similar to nerve injury rodent models $\mathrm{s}^{7,22}$ produced by ligation of branches of the sciatic nerve, except that only the common peroneal nerve was ligated in the sheep. 
A

Contralateral

B

Ipsilateral

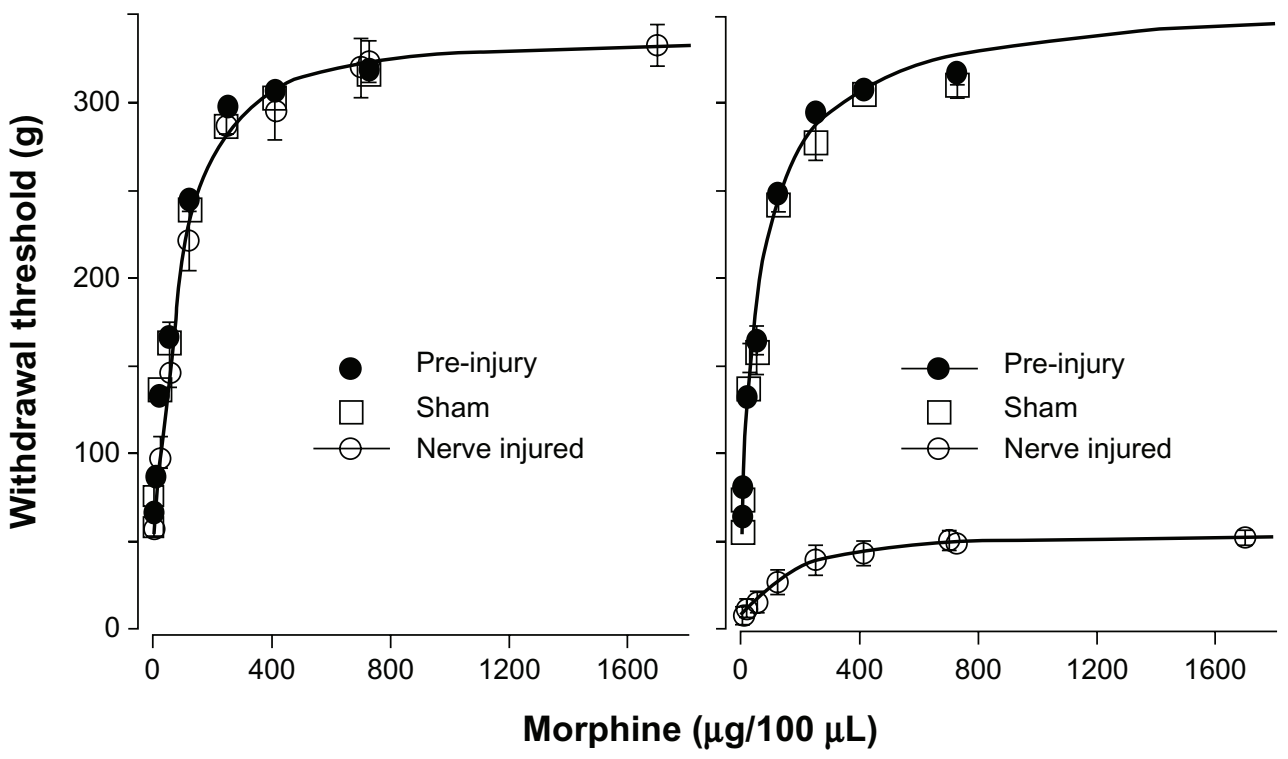

Figure 5 Dose-response of IT morphine on hindlimb withdrawal threshold. The hindlimb withdrawal threshold was determined 30 minutes after each IT dose of morphine. Each dose of morphine was given in $100 \mu \mathrm{L}$ volume. Pre-injury sheep were tested I-2 weeks before surgery. Sham and PNI sheep were tested 3 weeks after surgery. (A) Dose-response curve of pre-injury, sham, and nerve-injured contralateral hindlimbs. Dose-response curves were plotted as withdrawal threshold (g) versus cumulative morphine dose. The theoretical curves (solid lines) shown were fit to the contralateral nerve injured data with the Hill equation. The ED ${ }_{50}$ was determined from the fitted curve. The morphine $\mathrm{ED}_{50}$ for contralateral hindlimbs of pre-injury $(73.4 \mu \mathrm{g})$, sham $(74.8 \mu \mathrm{g})$, and nerve-injured sheep $(88.3 \mu \mathrm{g})$ were not significantly different. (B) Dose-response curve of pre-injury, sham, and nerve-injured ipsilateral hindlimbs. The ipsilateral pre-injury curve was extrapolated to the x-axis limit. The theoretical curves (solid lines) shown were fit to the ipsilateral pre-injury and ipsilateral nerve-injured data with the Hill equation.

Note: The $\mathrm{ED}_{50}$ was $76.3 \mu \mathrm{g}$ for pre-injury, $65.5 \mu \mathrm{g}$ for sham, and $159.0 \mu \mathrm{g}$ for nerve-injured sheep. Some of the standard errors of the data $\leq$ symbol sizes.

Abbreviations: $\mathrm{ED}_{50}$, the IT morphine dose that produces $50 \%$ of maximal analgesia; IT, intrathecal; PNI, peroneal nerve injury.

The injury resulted in a large reduction of mechanical withdrawal threshold on the ipsilateral limb 3 days after injury, and the allodynia was maintained for the duration of the study, ie, 8 weeks (Figure 3 ). Surgery alone did not change the hindlimb withdrawal threshold as evident by the lack of significant change between pre-injury and sham sheep. We further found that the dose-dependent analgesic effect of IT morphine of the contralateral limb of the PNI sheep is similar to that of ipsi- and contralateral limbs of pre-injury and sham sheep, but is very different from that of the ipsilateral limb of PNI sheep (Figure 5). In the contralateral hindlimb, IT morphine increased the withdrawal threshold from $57.4 \mathrm{~g}$ to $336.7 \mathrm{~g}$. In the ipsilateral hindlimb, morphine increased the withdrawal threshold from 9.6 to $54.8 \mathrm{~g}$ and thus almost completely reversed the allodynia ( $54.8 \mathrm{~g} / 57.4 \mathrm{~g})$. Unlike the contralateral hindlimb, morphine did not produce further antinociception beyond the allodynia range. The maximal withdrawal threshold produced by morphine in the ipsilateral limb was $16.2 \%(54.8 \mathrm{~g} / 336.7 \mathrm{~g})$ of that of the contralateral hindlimb. Thus, morphine is only partially effective in producing analgesia in the nerve injured limb. This result is consistent with clinical observations, based on a systematic review of randomized clinical trials, that opioids are effective in treating nociceptive pain yet only moderately effective in treating neuropathic pain patients. ${ }^{23}$

Comparisons of pain responses and morphine effects between patients and animal models have been difficult because of the extensive differences in how clinical and animal data are obtained. Most clinical pain studies rely on subjective patient reports of pain, whereas animal studies rely on reflexive measurements. For example, a clinical study based on patient questionnaires showed that spontaneous pain state was much more prevalent than the evoked thermal and mechanical hyperalgesia. ${ }^{24}$ Based on that study, many researchers have suggested that animal models do not accurately represent human pain. Other clinical studies measuring sensory thresholds in pain patients, a more objective measurement of pain, showed that (1) fibromyalgia patients displayed hypersensitivity to thermal and mechanical stimuli and (2) chronic back pain and nerve injury patients experienced pressure pain hypersensitivity. ${ }^{25,26}$ In a large (1236 patients) study of somatosensory abnormalities of neuropathic pain patients using quantitative sensory testing, including assessment of thresholds for mechanical and thermal stimuli, $92 \%$ of patients showed at least one quantitative sensory testing abnormality. ${ }^{27}$ Thus, unlike in the questionnaire study, 
stimulus-evoked allodynia and hyperalgesia are frequently observed in the study of sensory thresholds of pain patients.

In our study of duration of analgesic effect of IT morphine, morphine effect in pre-injury sheep was found to last for 3 hours (Figure 4), which is comparable to that in rats ( 2 hours), ${ }^{28}$ but is much shorter than the duration reported in clinical reviews and meta-analyses (12-24 hours). ${ }^{29,30}$ In clinical studies, duration was determined by indirect measurements, such as the time interval to request additional analgesia or the amount of additional analgesia. ${ }^{30}$ Without objective measurements of morphine-induced analgesia in patients, quantitative comparison of morphine analgesic duration between clinical and animal studies would not be feasible. These examples illustrate a need to conduct well-controlled sensory threshold studies in pain patients to facilitate the comparison between patients and animal models to effectively bridge the gap between self-reported pain scores of clinical studies and evoked sensory responses of animal studies.

A limitation of using sheep as a pain model is the lack of the respiratory sedative effects of IV opioids. ${ }^{31,32}$ We also observed a lack of respiratory sedation with IT morphine in sheep. Another limitation is that the ruminant digestive system may not accurately test oral drugs. However, the effect of opioids on gastrointestinal motility has been studied in sheep. ${ }^{33}$

There are apparent differences in nerve injury-induced withdrawal threshold and morphine responses of rats and sheep. In sheep, the contralateral hindlimb has similar nociceptive withdrawal threshold and morphine-induced analgesia as in pre-injury and sham sheep (Figure 3); therefore, the contralateral hindlimb can serve as the control. In the SNI and SNL rat models, the effect of nerve injury on the contralateral side has been controversial. Some researchers observed a concomitant reduction in contralateral withdrawal threshold after nerve injury ${ }^{5,34}$ while others did not. ${ }^{4,35}$ Changes in the expression of neuropeptides, receptor proteins, and ion channels in both the ipsilateral and contralateral nerves, which often occur after axotomy, may be responsible for these concomitant changes. ${ }^{36}$

We also compared morphine efficacies obtained in sheep with those estimated in humans and rats. A direct measurement of morphine efficacy is not feasible in clinical studies due to ethical limitations. The ceiling analgesic dose, which is defined as a dose that produces the greatest analgesic effect with tolerable side effects, was used to assess morphine efficacy. ${ }^{29}$ In a meta-analysis, 21 clinical studies measured analgesia and side effects of IT morphine (respiratory depression). The smallest dose producing significant respiratory depression in adult patients $(<70$ years) was the $560 \mu \mathrm{g}$ dose.$^{30}$ In four clinical studies, the $500 \mu \mathrm{g}$ dose resulted in no respiratory depression. ${ }^{37-40} \mathrm{We}$ therefore chose $500 \mu \mathrm{g}$ as the ceiling analgesic dose of IT morphine in humans (Table 1).

In animal studies, we defined the ceiling analgesic dose for IT morphine as the dose that produced the withdrawal threshold at $80 \%$ of $\mathrm{E}_{\max }$, which represents the transition from the linear to the plateau phase of the dose-response curve. In sheep, we found that IT morphine reached its ceiling effect at $300 \mu \mathrm{g}$ for sham ipsilateral, $250 \mu \mathrm{g}$ for nerve injured contralateral, and $385 \mu \mathrm{g}$ for nerve injured ipsilateral sheep (Figure 5 and Table 1). The morphine ceiling doses for rats calculated from published data were 1,1 , and $0.7 \mu \mathrm{g}$ for postoperative, SNL, and SNI rat models. respectively. ${ }^{4,5,16}$ Allometric scaling with a $3 / 4(0.75)$ power model describes the relationship between whole body metabolic rate and body weight/size and has been used successfully to predict morphine doses in humans of all ages. ${ }^{44}$ We therefore used the allometric scaling factor, which is defined as (human weight $[\mathrm{kg}] /$ animal weight $[\mathrm{kg}])^{0.75}$ to take into account the difference in the body size between human and sheep or rat and to estimate human equivalent morphine ceiling doses (animal ceiling dose $\times$ scaling factor). For sheep, the human equivalent ceiling doses are $504.5 \mu \mathrm{g}$ for sham, $420.3 \mu \mathrm{g}$ for nerve injury contralateral, and $647.6 \mu \mathrm{g}$ for nerve injury ipsilateral limb, which are within 16\%-30\% of the human morphine ceiling dose $(500 \mu \mathrm{g})$. For rats, the human equivalent ceiling doses are 55.0, 79.6, and $41.2 \mu \mathrm{g}$ for postoperative, SNL, and SNI rats, respectively, which are 6-12 times lower than the human morphine ceiling dose. ${ }^{41-43}$

Table I IT morphine ceiling dose comparison

\begin{tabular}{llll}
\hline Species & $\begin{array}{l}\text { Morphine } \\
\text { ceiling dose } \\
(\mu \mathbf{g})\end{array}$ & $\begin{array}{l}\text { Body } \\
\text { weight } \\
(\mathbf{k g})\end{array}$ & $\begin{array}{l}\text { Human equivalent } \\
\text { morphine ceiling } \\
\text { dose }(\mu \mathrm{g})\end{array}$ \\
\hline $\begin{array}{l}\text { Human (postop) } \\
\begin{array}{l}\text { Sheep (sham) } \\
\text { (contra or ipsi) }\end{array}\end{array}$ & 500 & 70 & 500.0 \\
$\begin{array}{l}\text { Sheep (nerve inj) } \\
\text { (contra) }\end{array}$ & 250 & 35 & 504.5 \\
$\begin{array}{l}\text { Sheep (nerve inj) } \\
\text { (ipsi) }\end{array}$ & 385 & 35 & 420.3 \\
$\begin{array}{l}\text { Rat (postop) } \\
\begin{array}{l}\text { Zahn et al }{ }^{41} \\
\text { Rat (SNL) }\end{array}\end{array}$ & 1 & 35 & 647.6 \\
$\begin{array}{l}\text { Zhang et al }{ }^{42} \\
\text { Rat (SNI) }\end{array}$ & 1 & 0.3 & 55.0 \\
Zhao et al ${ }^{43}$ & 0.7 & 0.225 & 79.6 \\
\hline
\end{tabular}

Note: Human equivalent morphine ceiling dose $=($ morphine ceiling dose $) \times\left(\mathrm{W}_{\text {human }} I\right.$ $\left.\mathrm{W}_{\text {animal }}\right)^{0.75}$.

Abbreviations: Postop, postoperative; contra, contralateral; ipsi, ipsilateral; inj, injury; SNL, spinal nerve ligation; SNI, spared nerve injury. 
It is of interest to determine the factors contributing to the large difference in estimated human equivalent ceiling dose between sheep and rats. The possible factors include differences in peripheral nerve injury (PNI versus SNL, or SNI) and/or the methods used in behavioral testing (limb versus paw withdrawal).

The PNI sheep model is a better model for estimating human equivalent morphine analgesic ceiling dose than nerve-injured rat models. This, together with its fast onset and stable and long-lasting pain behavioral characteristics, suggests that the PNI sheep model will be a useful tool for developing therapies of chronic pain and more accurately predicting drug dosage and delivery prior to clinical trials. We do not suggest replacing rat nerve injury models with the sheep PNI model, but a multispecies approach will improve the success rates of clinical trials.

\section{Acknowledgment}

The authors would like to thank Dr Christopher Suckow, Dr Monica Fann, Karolyn Niven, and Christine Kite for care of animals. This work was supported by the Foundation of Anesthesia and Education Research Mentored Research Training Grant, and National Institute of Health DA027360 (to D Wilkes) and NS30045 and DE17813 (to LYM Huang).

\section{Disclosure}

The authors report no conflicts of interest in this work.

\section{References}

1. Haanpaa M, Attal N, Backonja M, et al. NeuPSIG guidelines on neuropathic pain assessment. Pain. 2011;152(1):14-27.

2. Finnerup NB, Sindrup SH, Jensen TS. The evidence for pharmacological treatment of neuropathic pain. Pain. 2010;150(3):573-581.

3. Kola I, Landis J. Can the pharmaceutical industry reduce attrition rates? Nat Rev Drug Discov. 2004;3(8):711-715.

4. Decosterd I, Woolf CJ. Spared nerve injury: an animal model of persistent peripheral neuropathic pain. Pain. 2000;87(2):149-158.

5. Kim SH, Chung JM. An experimental model for peripheral neuropathy produced by segmental spinal nerve ligation in the rat. Pain. 1992;50(3): 355-363.

6. Bennett GJ, Xie YK. A peripheral mononeuropathy in rat that produces disorders of pain sensation like those seen in man. Pain. 1988;33(1): 87-107.

7. Lee BH, Won R, Baik EJ, Lee SH, Moon CH. An animal model of neuropathic pain employing injury to the sciatic nerve branches. Neuroreport. 2000;11(4):657-661.

8. Hassenbusch SJ, Satterfield WC, Gradert TL. A sheep model for continuous intrathecal infusion of test substances. Hum Exp Toxicol. 1999;18(2):82-87.

9. Scheerlinck JP, Snibson KJ, Bowles VM, Sutton P. Biomedical applications of sheep models: from asthma to vaccines. Trends Biotechnol. 2008;26(5):259-266.

10. Kijas JW, Lenstra JA, Hayes B, et al. Genome-wide analysis of the world's sheep breeds reveals high levels of historic mixture and strong recent selection. PLoS biology. 2012;10(2):e1001258.
11. Atanasov AT. The linear allometric relationship between total metabolic energy per life span and body mass of mammals. Bio Systems. 2007; 90(1):224-233.

12. Wilke HJ, Kettler A, Wenger KH, Claes LE. Anatomy of the sheep spine and its comparison to the human spine. Anat Rec. 1997;247(4): 542-555.

13. Nolan A, Livingston A, Morris R, Waterman A. Techniques for comparison of thermal and mechanical nociceptive stimuli in the sheep. J Pharmacol Methods. 1987;17(1):39-49.

14. Lebeaux M. Sheep: a model for testing spinal and epidural anesthetic agents. Lab Anim Sci. 1975;25(5):629-633.

15. Detweiler DJ, Eisenach JC, Tong C, Jackson C. A cholinergic interaction in alpha 2 adrenoceptor-mediated antinociception in sheep. J Pharmacol Exp Ther. 1993;265(2):536-542.

16. Mather LE, Cousins MJ, Huang YF, Pryor ME, Barratt SM. Lack of secondary hyperalgesia and central sensitization in an acute sheep model. Reg Anesth Pain Med. 2000;25(2):174-180.

17. Yaksh TL, Collins JG. Studies in animals should precede human use of spinally administered drugs. Anesthesiology. 1989;70(1):4-6.

18. Gradert TL, Baze WB, Satterfield WC, Hildebrand KR, Johansen MJ, Hassenbusch SJ. Safety of chronic intrathecal morphine infusion in a sheep model. Anesthesiology. 2003;99(1):188-198.

19. Ley SJ, Waterman AE, Livingston A. A field study of the effect of lameness on mechanical nociceptive thresholds in sheep. Vet Rec. 1995 ; 137(4):85-87.

20. Dolan S, Evans NP, Richter TA, Nolan AM. Expression of gonadotropin-releasing hormone and gonadotropin-releasing hormone receptor in sheep spinal cord. Neurosci Lett. 2003;346(1-2):120-122.

21. Duttaroy A, Kirtman R, Farrell F, et al. The effect of cumulative dosing on the analgesic potency of morphine in mice. Pharmacol Biochem Behav. 1997;58(1):67-71.

22. Amaya F, Decosterd I, Samad TA, et al. Diversity of expression of the sensory neuron-specific TTX-resistant voltage-gated sodium ion channels SNS and SNS2. Mol Cell Neurosci. 2000;15(4): 331-342.

23. Morlion B. Pharmacotherapy of low back pain: targeting nociceptive and neuropathic pain components. Curr Med Res Opin. 2011;27(1): 11-33.

24. Backonja MM, Stacey B. Neuropathic pain symptoms relative to overall pain rating. J Pain. 2004;5(9):491-497.

25. Gottrup H, Nielsen J, Arendt-Nielsen L, Jensen TS. The relationship between sensory thresholds and mechanical hyperalgesia in nerve injury. Pain. 1998;75(2-3):321-329.

26. Blumenstiel K, Gerhardt A, Rolke R, et al. Quantitative sensory testing profiles in chronic back pain are distinct from those in fibromyalgia. Clin J Pain. 2011;27(8):682-690.

27. Maier C, Baron R, Tolle TR, et al. Quantitative sensory testing in the German Research Network on Neuropathic Pain (DFNS): somatosensory abnormalities in 1236 patients with different neuropathic pain syndromes. Pain. 2010;150(3):439-450.

28. Penning JP, Yaksh TL. Interaction of intrathecal morphine with bupivacaine and lidocaine in the rat. Anesthesiology. 1992;77(6): 1186-2000.

29. Rathmell JP, Lair TR, Nauman B. The role of intrathecal drugs in the treatment of acute pain. Anesth Analg. 2005;101(Suppl 5):S30-S43.

30. Meylan N, Elia N, Lysakowski C, Tramer MR. Benefit and risk of intrathecal morphine without local anaesthetic in patients undergoing major surgery: meta-analysis of randomized trials. Br JAnaesth. 2009; 102(2):156-167.

31. Waterman AE, Livingston A, Amin A. Further studies on the antinociceptive activity and respiratory effects of buprenorphine in sheep. J Vet Pharmacol Ther. 1991;14(3):230-234.

32. Waterman AE, Livingston A, Amin A. Analgesic activity and respiratory effects of butorphanol in sheep. Res Vet Sci. 1991;51(1):19-23.

33. Ruckebusch Y, Bardon T, Pairet M. Opioid control of the ruminant stomach motility: functional importance of mu, kappa and delta receptors. Life Sci. 1984;35(17):1731-1738. 
34. Erichsen HK, Blackburn-Munro G. Pharmacological characterisation of the spared nerve injury model of neuropathic pain. Pain. 2002; 98(1-2):151-161.

35. Scott DA, Wright CE, Angus JA. Actions of intrathecal omegaconotoxins CVID, GVIA, MVIIA, and morphine in acute and neuropathic pain in the rat. Eur J Pharmacol. 2002;451(3):279-286.

36. Koltzenburg M, Wall PD, McMahon SB. Does the right side know what the left is doing? Trends Neurosci. 1999;22(3):122-127.

37. Alhashemi JA, Sharpe MD, Harris CL, Sherman V, Boyd D. Effect of subarachnoid morphine administration on extubation time after coronary artery bypass graft surgery. J Cardiothorac Vasc Anesth. 2000;14(6): 639-644.

38. El-Hakeem. Effect of subarachnoid morphine administration on haemodynamic stressand extubation time after valve replacement surgery. Egypt J Anaesth. 2003;19:9-19.

39. Liu N, Kuhlman G, Dalibon N, Moutafis M, Levron JC, Fischler M. A randomized, double-blinded comparison of intrathecal morphine, sufentanil and their combination versus IV morphine patient-controlled analgesia for postthoracotomy pain. Anesth Analg. 2001;92(1): $31-36$.
40. Sarma VJ, Boström UV. Intrathecal morphine for the relief of posthysterectomy pain - a double-blind, dose-response study. Acta Anaesthesiol Scand. 1993;37(2):223-227.

41. Zahn PK, Gysbers D, Brennan TJ. Effect of systemic and intrathecal morphine in a rat model of postoperative pain. Anesthesiology. 1997; 86(5):1066-1077.

42. Zhang Y, Conklin DR, Li X, Eisenach JC. Intrathecal morphine reduces allodynia after peripheral nerve injury in rats via activation of a spinal A1 adenosine receptor. Anesthesiology. 2005;102(2):416-420.

43. Zhao C, Tall JM, Meyer RA, Raja SN. Antiallodynic effects of systemic and intrathecal morphine in the spared nerve injury model of neuropathic pain in rats. Anesthesiology. 2004;100(4):905-911.

44. Holford NH, Ma SC, Anderson BJ. Prediction of morphine dose in humans. Paediatr Anaesthes. 2012;22(3):209-222.
Journal of Pain Research

\section{Publish your work in this journal}

The Journal of Pain Research is an international, peer-reviewed, open access, online journal that welcomes laboratory and clinical findings in the fields of pain research and the prevention and management of pain. Original research, reviews, symposium reports, hypothesis formation and commentaries are all considered for publication.

\section{Dovepress}

The manuscript management system is completely online and includes a very quick and fair peer-review system, which is all easy to use. Visit http://www.dovepress.com/testimonials.php to read real quotes from published authors. 